\title{
Pattern of Recurrence of Myxofibrosarcoma is not Associated with Pattern at Presentation or Rate of Delayed Diagnosis
}

\author{
Corey Daniels, ${ }^{1}$ Wei-Lien Wang, ${ }^{2}$ John Edward Madewell, ${ }^{1}$ Wei Wei, ${ }^{3}$ and Behrang Amini1, ${ }^{1,}$ \\ ${ }^{1}$ The University of Texas MD Anderson Cancer Center, Texas, United States of America \\ ${ }^{2}$ Department of Pathology, The University of Texas MD Anderson Cancer Center, Texas, United States of America \\ ${ }^{3}$ Department of Biostatistics, The University of Texas M.D. Anderson Cancer Center, United States of America \\ "Corresponding author: Behrang Amini, 1400 Pressler St, Unit 1475,Houston, TX 77030, United States of America. Tel: +1-7137925181, Fax: +1-7135636633, E-mail: \\ bamini@mdanderson.org
}

Received 2015 August 20; Revised 2015 October 22; Accepted 2016 January 02.

\begin{abstract}
Background: The tendency of myxofibrosarcoma (MFS) to have a non-nodular appearance on magnetic resonance imaging (MRI) may present a challenge in detection of local recurrence. Appropriate index of suspicion of expected imaging appearance can alleviate this challenge.

Objectives: Our objectives were to determine if: 1) imaging pattern at recurrence is associated with delayed diagnosis, and 2) appearance at recurrence can be predicted based on appearance at presentation.

Patients and Methods: A retrospective cohort study was performed. Two analysis groups were used: patients with recurrence captured on MRI (objective 1) and patients with MRI prior to resection and at recurrence (objective 2). Three radiologists scored images independently and in consensus as infiltrative (tail-like spread), focal (absence of spread), or mixed. Consensus results were used for analysis with Fisher's exact test.

Results: There was substantial agreement among the 3 readers $(k=0.72,95 \%$ confidence interval: 0.59 - 0.85$)$. Half of all infiltrative cases were associated with a delay in diagnosis, compared to $10 \%$ of mixed or focal cases; however, there was no statistically significant association between infiltrative pattern at recurrence and delayed diagnosis $(P=0.08)$. In $70 \%$ of cases, recurrence had the same appearance as presentation, suggesting a trend; however, there was no association between appearance at baseline and recurrence $(\mathrm{P}=0.1)$.

Conclusion: Imaging appearance at baseline does not reliably predict imaging appearance at recurrence. Therefore, a high index of suspicion for the infiltrative pattern is required in assessment of post-operative MRIs in patients with MFS.
\end{abstract}

Keywords: Sarcoma, MRI, Recurrence

\section{Background}

Myxofibrosarcoma (MFS, previously myxoid malignant fibrous histiocytoma) is a soft-tissue sarcoma of fibroblastic origin with myxoid matrix that affects patients in the sixth to eighth decades of life (1-3). Typical imaging features of MFS have been described $(4,5)$ and can be seen in Figure 1. The lesions have a heterogeneous appearance on T1-and T2-weighted images (WI) and can have ill-defined infiltrative margins (more below). On T1-WI, the lesions are typically isointense to muscle, but can have areas of mild hyperintensity. On T2-WI, MFS has high signal owing to its myxoid content and can have fluid-fluid levels. Postcontrast images reveal heterogeneous enhancement with variable peripheral nodular enhancement and the featherlike enhancement typically seen with myxoid neoplasms.

MFS has a propensity for repeated local recurrence, even after wide excision $(3,6)$, with local recurrence rates of up to $79 \%$ (7-9). The high rate of local recurrence is thought to be related to the infiltrative nature of these tumors (10-
12), which can be reflected in their unusual appearance on magnetic resonance imaging (MRI): In contrast to the focal and mass-like appearance of most soft tissue sarcomas (13), MFS can present as an infiltrative lesion with variable presence of a well-defined mass $(4,9,14)$. This range of imaging appearance can be classified as infiltrative, focal, or mixed (Figure 1).

In our experience, the infiltrative pattern has presented a challenge in detection of recurrent disease following surgery because of its similarity to post-operative inflammation and the expectation of radiologists for nodular enhancement to indicate recurrence. However, the association of imaging appearance with delayed diagnosis of recurrence has not been systematically studied (objective 1 , see below).

The difficulty in detection of recurrent disease may be alleviated if the radiologist could adjust her index of suspicion for recurrence based on the imaging appearance at presentation. While it is thought that imaging appearance at recurrence follows that of the primary tumor (5), this as- 

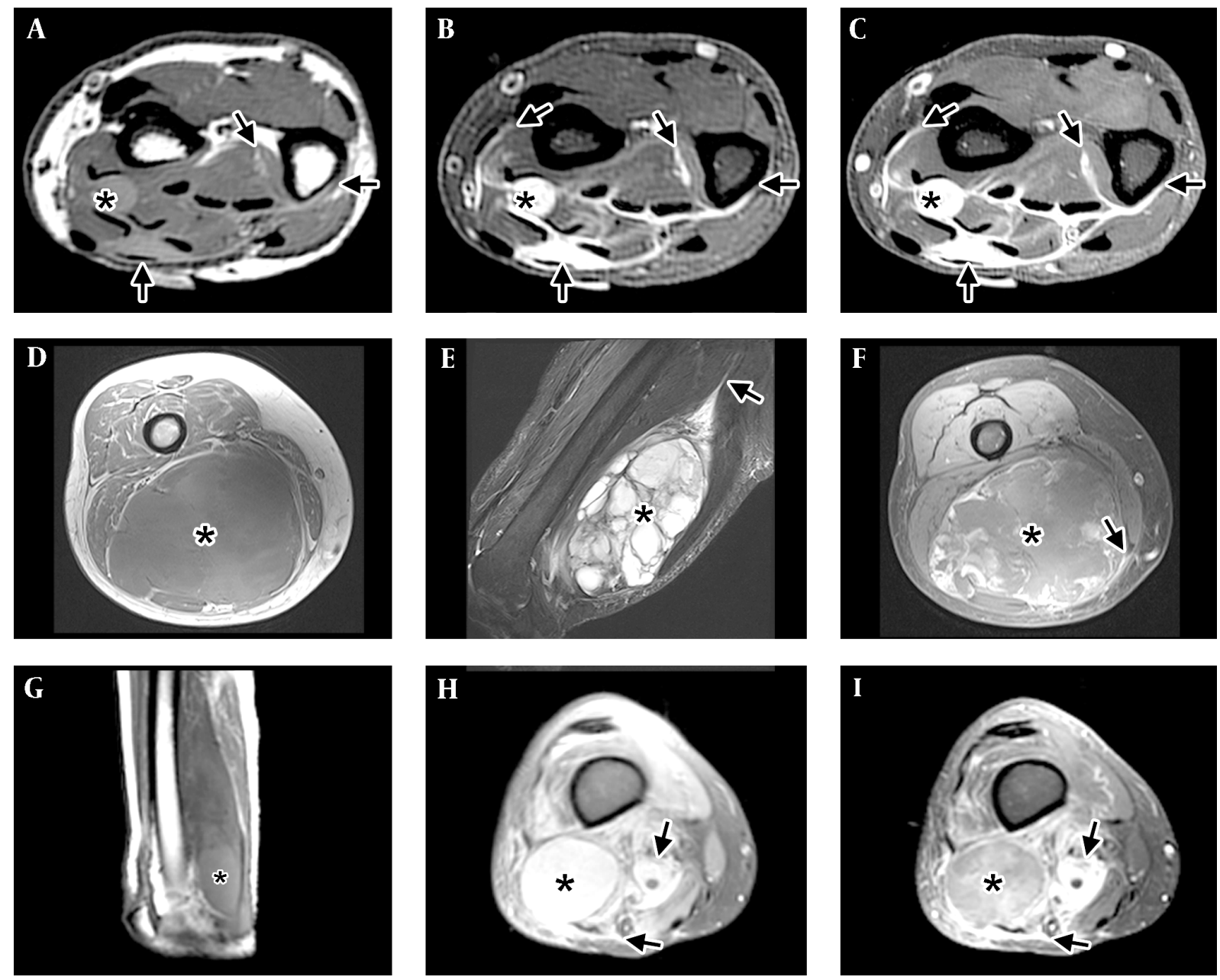

Figure 1. Imaging patterns of myxofibrosarcoma (MFS). A-C, Infiltrative pattern in the distal forearm, with tail-like multidirectional spread along fascial planes (arrows) beyond the tiny nodule $\left({ }^{*}\right)$. A, Short-axis T1-WI shows the mass has intermediate T1 signal, hyperintense to muscle. Tail-like extensions (arrows) can be faintly seen. B, Short-axis T2-WI with fat suppression shows the lesion to have high signal, close to that expected for fluid. The tail-like extensions can be seen more clearly. C, Short-axis post-contrast T1-WI with fat suppression shows the mass and the tail-like extensions to enhance avidly. D-F, Focal mass $\left(^{*}\right)$ in the distal thigh, with minimal to no spread along fascial planes (arrow) and good demarcation of the tumor by a thin rim. D, Axial T1-WI shows an intermediate signal mass $\left({ }^{*}\right)$ with areas of subtle increased signal compared to muscle located in the posterior compartment of the thigh. Tail-like extension is not well seen. E, Sagittal T2-WI with fat suppression shows the dominant multiloculated mass $\left({ }^{*}\right)$ with high signal internally and multiple low-signal septations. A long tail (arrow) can be seen extending proximally. F, Axial post-contrast T1-WI with fat suppression shows a mass $\left(^{*}\right)$ with peripheral nodular and central feather-like pattern of enhancement typically seen with myxoid neoplasms. A small lateral tail can be seen laterally. G-I, Mixed pattern, with a dominant mass $\left({ }^{*}\right)$ and extensive surrounding infiltration (arrows). G, Sagittal T1-WI shows a mass in the posterior compartment of the thigh with a higher signal component distally $(*)$. H, Axial PD fat suppressed image at the distal thigh shows a high-signal mass $\left({ }^{*}\right)$ and areas of extension (arrows) in the adjacent tissues. I, Axial T1-WI with fat suppression shows a mass $\left({ }^{*}\right)$ with central feather-like pattern of enhancement typically seen with myxoid neoplasms and enhancing infiltration (arrows) of the adjacent tissues.

sociation has not been systematically studied, and the reported association remains anecdotal (objective 2, see below).

Our central hypotheses are that 1) The infiltrative pattern at recurrence is associated with a higher rate of delayed diagnosis than the focal or mixed patterns, and 2) The imaging appearance at recurrence can be predicted based on the appearance at presentation.

\section{Objectives}

As noted above, the objectives of this study are:

1) To determine the association of imaging appearance at time of recurrence with the rate of delayed diagnosis.

2) To determine whether the imaging appearance at baseline can reliably predict the imaging appearance at recurrence. 


\section{Patients and Methods}

Waiver of consent was obtained from the institutional review board as mandated by institutional policy for this retrospective cohort study. The study protocol conformed to the ethical guidelines of the 1975 Declaration of Helsinki (15) as reflected in a priori approval by our institution's human research committee/institutional review board. Two hundred forty six patients with a suspected diagnosis of MFS or myxoid malignant fibrous histiocytoma were identified through search of the radiology and pathology databases $(16,17)$. Inclusion criteria included confirmed diagnosis of MFS after review by a sarcoma pathologist (WLW), local control with surgery (excluding amputation), and presence of recurrent disease on no earlier than the third post-operative MRI. This last criterion was required in order to ensure that a missed diagnosis could be reasonably quantified. For example, it would not be reasonable to conclude that recurrence was missed on the first postoperative MRI, since it is usually not possible to separate recurrent disease from expected inflammation, even in the case of typical soft tissue sarcomas with a nodular appearance. In addition, while it would be possible to detect recurrence on the second post-operative MRI, it would not be possible to determine if the lesion was present on the prior study in order to determine that a delay in diagnosis had occurred.

\subsection{Data Collection}

The following data were obtained from the electronic medical record: length of follow-up, treatment modality (e.g., surgery, radiation, chemotherapy), and reported date of recurrence. Start time for determination of time to recurrence was defined as surgery date or end date of postoperative radiation therapy, whichever occurred later. The final diagnosis, tumor grade, and surgical margins were obtained by review of the pathology reports and, when necessary, the histopathological slides.

\subsection{Image Acquisition and Analysis}

Images were acquired on a variety of scanners between 2002 and 2014, all at 1.5-T field strength. The majority of MRIs were performed in-house on GE Signa scanners (GE Healthcare, Madison, USA). Images obtained at outside facilities were imported into the local PACS for analysis. These outside scans were obtained on GE Signa, Siemens Trio, Avanto, and Symphony (Siemens Healthcare, Malvern, USA), Philips Intera (Philips Healthcare, Best, The Netherlands), and Marconi Eclipse (London, UK).

Images were reviewed by two readers (CD and $B A$ ) in consensus to determine if a lesion met inclusion criteria.
Images prior to the reported date of recurrence were reviewed to determine the true date of recurrence. Local recurrence was defined as disease within a $5 \mathrm{~cm}$ shell of the resection bed. Growth of gross residual disease after surgery and radiation therapy was not considered local recurrence. In cases where symptomatic local recurrence was not prospectively detected on MRI, the date the contradiction was resolved was used as the time of recurrence. In cases where asymptomatic local recurrence was missed on MRI but detected on another modality (e.g., positron emission tomography), a conservative estimate was made that the recurrence would have been detected at the next scheduled MRI based on the post-operative follow-up pattern for soft tissue sarcomas at our institution (every 3 months for 2 years; every 4 months for 1 year; every 6 months for 2 years; once a year for 5 years; then as needed) (18).

Patients that met inclusion criteria were divided into two groups (Table 1). Group 1 consisted of patients with recurrent disease captured on MRI and was used to assess the association between imaging pattern at recurrence with delay in diagnosis (objective 1). If multiple recurrences occurred, each recurrence captured on MRI was included for analysis. Group 2 consisted of patients with recurrent disease captured on MRI and the availability of pre-therapy MRI. This group was used to assess the association between imaging appearance at presentation and at recurrence (objective 2). If there were multiple recurrences, only the first recurrence was selected for analysis in this group.

A training set of images was prepared by a musculoskeletal attending radiologist (BA) from images not used in either test group. This studies used for analysis were first randomized (using microsoft excel's rand function (19) and then rearranged to ensure that studies from the same patient at different time points (i.e., at presentation and recurrence) did not appear in close sequence in order to reduce the memory effect present in repeated radiologic observations (20). Three readers, two musculoskeletal attending radiologists (BA and JEM) and one musculoskeletal fellow (CD) then scored the lesions. This was done independently first, and then in consensus. The imaging pattern was classified as infiltrative (tail-like multidirectional spread along fascial planes), focal (absence of spread and good demarcation of the tumor by a thin rim), or mixed (Figure 1) as modified form (14).

\subsection{Statistical Analysis}

Statistical analysis was performed using R software package for windows (21). The consensus scores for the patients was used for analysis. Fisher's exact test (22) was used to assess the association between imaging pattern at presentation and recurrence and the association between delay in diagnosis and imaging pattern at recurrence. Inter- 


\begin{tabular}{|c|c|c|c|c|c|}
\hline Patient ID & MRI ID & Age & Sex & 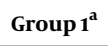 & Group $2^{b}$ \\
\hline \multirow[t]{2}{*}{5} & 1 & 59 & M & $\mathrm{Y}$ & \\
\hline & 2 & & & $\mathrm{Y}$ & \\
\hline \multirow[t]{2}{*}{7} & 3 & 51 & $\mathrm{~F}$ & $\mathrm{Y}$ & \\
\hline & 4 & & & $\mathrm{Y}$ & \\
\hline 10 & 5 & 53 & M & $\mathrm{Y}$ & \\
\hline 13 & 6 & 46 & $\mathrm{~F}$ & $\mathrm{Y}$ & \\
\hline 19 & 7 & 46 & $\mathrm{~F}$ & $\mathrm{Y}$ & \\
\hline 22 & 8,9 & 65 & M & & $\mathrm{Y}$ \\
\hline \multirow[t]{3}{*}{23} & 10,11 & 72 & $\mathrm{~F}$ & $\mathrm{Y}$ & $\mathrm{Y}$ \\
\hline & 12 & & & $\mathrm{Y}$ & \\
\hline & 13 & & & Y & \\
\hline 35 & 14 & 51 & M & Y & \\
\hline 42 & 15,16 & 69 & $\mathrm{~F}$ & $\mathrm{Y}$ & $\mathrm{Y}$ \\
\hline 45 & 17,18 & 60 & F & $\mathrm{Y}$ & $\mathrm{Y}$ \\
\hline 46 & 19,20 & 82 & $\mathrm{~F}$ & Y & Y \\
\hline 53 & 21 & 85 & M & $\mathrm{Y}$ & \\
\hline 61 & 22,23 & 79 & $\mathrm{~F}$ & & $\mathrm{Y}$ \\
\hline 72 & 24 & 67 & M & $\mathrm{Y}$ & \\
\hline \multirow[t]{2}{*}{77} & 25,26 & 55 & $\mathrm{~F}$ & $\mathrm{Y}$ & $\mathrm{Y}$ \\
\hline & 27 & & & Y & \\
\hline 94 & 28,29 & 73 & M & & $\mathrm{Y}$ \\
\hline 100 & 30,31 & 80 & M & & $\mathrm{Y}$ \\
\hline 102 & 32,33 & 52 & M & Y & Y \\
\hline 103 & 34,35 & 44 & $\mathrm{~F}$ & $\mathrm{Y}$ & $\mathrm{Y}$ \\
\hline 113 & 36,37 & 47 & M & $\mathrm{Y}$ & $\mathrm{Y}$ \\
\hline 114 & 38 & 73 & M & $\mathrm{Y}$ & \\
\hline 117 & 39,40 & 78 & $\mathrm{~F}$ & $\mathrm{Y}$ & $\mathrm{Y}$ \\
\hline 136 & 41 & 82 & M & $\mathrm{Y}$ & \\
\hline
\end{tabular}

Abbreviations: M, male F, female MRI, magnetic resonance imaging.

${ }^{a}$ Patients in group 1 had at least one MRI (at recurrence), with or without MRI prior to surgery.

${ }^{\mathrm{b}}$ Patients in group 2 had 2 MRIs: at presentation (prior to surgery) and at recurrence.

observer agreement was assessed using kappa statistics (23). For the purposes of determining positive and negative predictive values (PPV and NPV, respectively) and positive and negative likelihood ratios (LR+ and LR-, respectively), infiltrative lesions were compared to the combined set of mixed/focal lesions. The reference standard was longitudinal follow-up.

\section{Results}

A total of 41 MRI studies in 23 patients (Table 1) were used in the final analyses. The imaging pattern was assessed individually and then in consensus by three readers (CD, JEM, and BA) and scored as infiltrative, focal, or mixed (Figure 1). There was substantial inter-observer agreement (Kappa $=0.72 ; 95 \%$ confidence interval (CI): 0.59 - 0.85).

Analysis of group 1 was performed next to determine association of imaging pattern at recurrence with delay in diagnosis. There were 24 recurrences in 20 patients (14 in- 
filtrative, 6 mixed, and 4 focal). Delay in diagnosis ranged from 90 to 218 days and was seen in 8 cases. Half (7) of the 14 infiltrative recurrences had a delay in diagnosis with a median of 92 days. None of the 6 mixed cases had a delay in diagnosis, and 1 out of the 4 focal recurrences was associated with a delay in diagnosis (105 days). The majority (88\%) of cases associated with a delay in diagnosis had the infiltrative pattern; however, there was only a trend for association between imaging pattern at recurrence and the presence of a delay in diagnosis $(\mathrm{P}=0.08)$.

Analysis of group 2 was performed next in order to determine association of imaging pattern at presentation and recurrence. Out of the 13 patients in this group, 9 (70\%) had the same imaging appearance at presentation and recurrence ( 3 infiltrative, 4 mixed, and 2 focal); however, there was no significant association between imaging pattern at presentation and recurrence $(\mathrm{P}=0.1)$.

All 3 infiltrative cases recurred as infiltrative, with a PPV of $100 \%$ for the infiltrative pattern (LR+ could not be calculated). One of the 3 focal cases recurred as infiltrative, and 2 of the 7 mixed cases recurred as infiltrative, with a NPV of $70 \%$ and LR- of 0.50 (95\% CI: $0.22-1.11$ ) for the infiltrative pattern.

\section{Discussion}

Our anecdotal experience prior to this investigation suggested that the tendency of MFS to recur with an infiltrative pattern (which mimics post-operative inflammation) would present a challenge in interpretation and lead to an increase in delayed diagnosis. In addition, it has been suggested that the imaging pattern at presentation would predict the imaging pattern at recurrence (5). If true, this would provide radiologists with an easy way to adjust their threshold for separating recurrent disease from postoperative inflammation.

The first objective of our study was to determine if our impression of the difficulty in interpreting these studies was supported by evidence of an increase in misdiagnosis rate associated with the infiltrative pattern of recurrence. The second objective of our study was to determine if the imaging pattern at recurrence was associated with the imaging pattern at presentation.

Regarding objective 1 , our results showed a trend for association between the infiltrative pattern and a delay in diagnosis of local recurrence. Exactly half of the 14 infiltrative recurrences were not prospectively diagnosed (compared to only $10 \%$ of the focal or mixed lesions). In addition 7 out of the 8 cases associated with a delayed diagnosis of recurrence had the infiltrative pattern at recurrence. However, no significant difference was found in the presence of a delay in diagnosis when infiltrative lesions were compared to the mixed and focal lesions $(\mathrm{P}=0.08)$. It must be noted, however, that this result may have been affected by the trepidation of interpreting radiologists at our institution when faced with post-operative MRIs of patients with MFS, and may not apply to other institutions that do not have the same experience in the follow-up of these lesions.

Regarding objective 2, our results suggest that while an infiltrative pattern at presentation was always associated with an infiltrative pattern at recurrence ( $P P V=100 \%)$, the inverse was not true (i.e., the focal or mixed lesions did not reliably recur as focal or mixed). Indeed the NPV for an infiltrative pattern at recurrence based on the pattern at presentation was only $70 \%$ with a poor LR- of 0.5 . Therefore, a high index of suspicion is warranted for areas of linear signal abnormality on post-operative MRIs regardless of the imaging appearance at presentation. This recommendation is obviously challenging to implement on immediate post-operative MRI; however, persistent or growing areas of linear signal abnormality on subsequent MRIs should not be dismissed as inflammation or granulation in patients with MFS.

Our study has several limitations. The most important issue is inherent to its retrospective design, which will inevitably lead to selection bias (24) and treatment and follow-up heterogeneity. Unfortunately, a prospective study for rare malignancies such as MFS would be impractical, and imaging-only studies would have difficulties recruiting patients due to absence of direct benefit to patients (25).

Another important limitation is the small size of our study. Even though 246 patients with a preliminary diagnosis of MFS were identified through keyword searches of radiology and pathology databases, less than $10 \%$ were suitable for final analysis due to failure to meet inclusion criteria. These included incorrect reported pathological diagnosis on radiology reports or due to changes in terminology and classification and absence of pre-therapy MRI (e.g., in the setting of unplanned excision).

\section{Acknowledgments}

None declared.

\section{Footnotes}

Authors' Contribution: Study concept and design: Behrang Amini; Acquisition of data: Corey Daniels, John Edward Madewell, Behrang Amini; Analysis and interpretation of data: Behrang Amini, Wei-Lien Wang; Drafting of the manuscript: Corey Daniels, Behrang Amini; Critical revision of the manuscript for important intellectual 
content: Behrang Amini, John Edward Madewell, Wei-Lien Wang, Corey Daniels; Statistical analysis: Wei Wei; Administrative, technical, and material support: Behrang Amini; Study supervision: Behrang Amini, John Edward Madewell.

Financial Disclosure: The authors have no relevant financial disclosures.

Funding/Support: This work was supported in part by the cancer center support grant (NCI Grant P30 CA016672).

\section{References}

1. Mentzel T, Van den berg E, Molenaar WM. Myxofibrosarcoma, In: Fletcher CDM, Unni KK, Mertens F, editors. Pathology and genetics of tumours of soft tissue and bone. Lyon: IARC Press; 2002.

2. Mentzel T, Calonje E, Wadden C, Camplejohn RS, Beham A, Smith MA, et al. Myxofibrosarcoma. Clinicopathologic analysis of 75 cases with emphasis on the low-grade variant. Am J Surg Pathol. 1996;20(4):391405. [PubMed: 8604805].

3. Weiss SW, Enzinger FM. Myxoid variant of malignant fibrous histiocytoma. Cancer. 1977;39(4):1672-85. [PubMed: 192434].

4. Lefkowitz RA, Landa J, Hwang S, Zabor EC, Moskowitz CS, Agaram $\mathrm{NP}$, et al. Myxofibrosarcoma: prevalence and diagnostic value of the "tail sign" on magnetic resonance imaging. Skeletal Radiol. 2013;42(6):809-18. doi: 10.1007/s00256-012-1563-6. [PubMed: 23318907].

5. Baheti AD, Tirumani SH, Rosenthal MH, Howard SA, Shinagare AB, Ramaiya $\mathrm{NH}$, et al. Myxoid soft-tissue neoplasms: comprehensive update of the taxonomy and MRI features. AJR Am J Roentgenol. 2015;204(2):374-85. doi: 10.2214/AJR.14.12888. [PubMed: 25615761].

6. Huang HY, Lal P, Qin J, Brennan MF, Antonescu CR. Low-grade myxofibrosarcoma: a clinicopathologic analysis of 49 cases treated at a single institution with simultaneous assessment of the efficacy of 3-tier and 4-tier grading systems. Hum Pathol. 2004;35(5):612-21. [PubMed: 15138937].

7. Merck C, Angervall L, Kindblom LG, Oden A. Myxofibrosarcoma. A malignant soft tissue tumor of fibroblastic-histiocytic origin. A clinicopathologic and prognostic study of 110 cases using multivariate analysis. Acta Pathol Microbiol Immunol Scand Suppl. 1983;282:1-40. [PubMed: 6444190].

8. Daniels J, Green CM, Freemont A, Paul A. The management of myxofibrosarcoma - a ten-year experience in a single specialist centre. Acta Orthop Belg. 2014;80(3):436-41. [PubMed: 26280619].

9. Kikuta K, Kubota D, Yoshida A, Morioka H, Toyama Y, Chuuman H, et al. An analysis of factors related to the tail-like pattern of myxofibrosarcoma seen on MRI. Skeletal Radiol. 2015;44(1):55-62. doi: 10.1007/s00256-014-1992-5. [PubMed: 25172220].
10. Manoso MW, Pratt J, Healey JH, Boland PJ, Athanasian EA. Infiltrative MRI pattern and incomplete initial surgery compromise local control of myxofibrosarcoma. Clin Orthop Relat Res. 2006;450:89-94. doi 10.1097/01.blo.0000229292.98850.14. [PubMed:16801862].

11. Yoo HJ, Hong SH, Kang Y, Choi JY, Moon KC, Kim HS, et al. MR imaging of myxofibrosarcoma and undifferentiated sarcoma with emphasis on tail sign; diagnostic and prognostic value. Eur Radiol. 2014;24(8):1749-57. doi: 10.1007/s00330-014-3181-2. [PubMed: 24889995].

12. Iwata S, Yonemoto T, Araki A, Ikebe D, Kamoda H, Hagiwara Y, et al. Impact of infiltrative growth on the outcome of patients with undifferentiated pleomorphic sarcoma and myxofibrosarcoma. J Surg Oncol. 2014;110(6):707-11. doi:10.1002/jso.23708. [PubMed: 24975462].

13. Amini B, Jessop AC, Ganeshan DM, Tseng WW, Madewell JE. Contemporary imaging of soft tissue sarcomas. J Surg Oncol. 2015;111(5):496-503. doi: 10.1002/jso.23801. [PubMed: 25348367].

14. Kaya M, Wada T, Nagoya S, Sasaki M, Matsumura T, Yamaguchi T, et al. MRI and histological evaluation of the infiltrative growth pattern of myxofibrosarcoma. Skeletal Radiol. 2008;37(12):1085-90. doi: 10.1007/s00256-008-0542-4. [PubMed: 18629459].

15. 29th WMA General Assembly. WMA Declaration of Helsinki - Ethical Principles for Medical Research Involving Human Subjects. Japan: World Medical Association; 1975.

16. Montage Healthcare Solutions . MONTAGE ${ }^{\mathrm{TM}}$ Search and Analytics. Philadelphia: Princeton Architectural; 2015.

17. Garg N. Garglets Toolkit. Houston: TX; 2015. Available at : http:// garglab.com.

18. Tseng WW, Amini B, Madewell JE. Follow-up of the soft tissue sarcoma patient.JSurg Oncol. 2015;111(5):641-5. doi:10.1002/jso.23814. [PubMed: 25322963].

19. Microsoft . Microsoft Excel. Redmond: WA;2013.

20. Ryan JT, Haygood TM, Yamal JM, Evanoff M, O'Sullivan P, McEntee M, et al. The "memory effect" for repeated radiologic observations. AJR Am J Roentgenol. 2011;197(6):W985-91. doi: 10.2214/AJR.10.5859. [PubMed: 22109344].

21. R Development Core Team R . A language and environment for statistical computing. Vienna: R Foundation for Statistical Computing 2015

22. Fisher RA. On the Interpretation of $\chi 2$ from Contingency Tables, and the Calculation of P. J Royal Stat Soci. 1922;85(1):87. doi: $10.2307 / 2340521$.

23. Cohen J. A Coefficient of Agreement for Nominal Scales. Educ Psychol Meas. 1960;20(1):37-46. doi: 10.1177/001316446002000104.

24. Sica GT. Bias in research studies. Radiology. 2006;238(3):780-9. doi 10.1148/radiol.2383041109. [PubMed: 16505391].

25. Kurland BF, Gerstner ER, Mountz JM, Schwartz LH, Ryan CW, Graham MM, et al. Promise and pitfalls of quantitative imaging in on cology clinical trials. Magn Reson Imaging. 2012;30(9):1301-12. doi: 10.1016/j.mri.2012.06.009. [PubMed: 22898682] 